\title{
Optimisation of variables for studying dilepton transverse momentum distributions at hadron colliders
}

\author{
A. Banfi ${ }^{1}$, S. Redford ${ }^{2}$, M. Vesterinen ${ }^{2}$, P. Waller ${ }^{2}$, T.R. Wyatt ${ }^{2, a}$ \\ ${ }^{1}$ Institute for Theoretical Physics, ETH Zurich, 8093 Zurich, Switzerland \\ ${ }^{2}$ Particle Physics Group, School of Physics and Astronomy, University of Manchester, Manchester M13 9PL, UK
}

Received: 9 September 2010 / Revised: 17 February 2011 / Published online: 10 March 2011

(C) The Author(s) 2011. This article is published with open access at Springerlink.com

\begin{abstract}
In future measurements of the dilepton $\left(Z / \gamma^{*}\right)$ transverse momentum, $Q_{T}$, at both the Tevatron and LHC, the achievable bin widths and the ultimate precision of the measurements will be limited by experimental resolution rather than by the available event statistics. In a recent paper the variable $a_{T}$, which corresponds to the component of $Q_{T}$ that is transverse to the dilepton thrust axis, has been studied in this regard. In the region, $Q_{T}<30 \mathrm{GeV}, a_{T}$ has been shown to be less susceptible to experimental resolution and efficiency effects than the $Q_{T}$. Extending over all $Q_{T}$, we now demonstrate that dividing $a_{T}$ (or $Q_{T}$ ) by the measured dilepton invariant mass further improves the resolution. In addition, we propose a new variable, $\phi_{\eta}^{*}$, that is determined exclusively from the measured lepton directions; this is even more precisely determined experimentally than the above variables and is similarly sensitive to the $Q_{T}$. The greater precision achievable using such variables will enable more stringent tests of QCD and tighter constraints on Monte Carlo event generator tunes.
\end{abstract}

\section{Introduction}

The production of $Z / \gamma^{*} \rightarrow e^{+} e^{-}$and $Z / \gamma^{*} \rightarrow \mu^{+} \mu^{-}$at hadron colliders provides an ideal testing ground for the predictions of QCD, due to the colourless and relatively background free final state. The dilepton transverse momentum, $Q_{T}$, distribution probes QCD radiation in the initial state. At high values of $Q_{T}\left(Q_{T}>30 \mathrm{GeV}\right.$, say), the fixed order perturbative calculations now available at NNLO $[1,2]$ are expected to yield accurate predictions. At low $Q_{T}$, soft gluon resummation techniques are required [3], with additional non-perturbative form factors determined in global fits to experimental data such as in [4]. Various event generators

a e-mail: twyatt@fnal.gov are also available [5-8], matching tree level matrix elements to parton showers tuned to data. Validation and tuning (form factors and parton showers) of these models require comparison with experimental data that have been corrected for detector resolution and efficiency effects. Improved understanding of these production models will increase sensitivity to new physics signals at hadron colliders.

The $Q_{T}$ distribution has been measured at the Fermilab Tevatron, by the CDF [9] and DØ [10-13] Collaborations. The most recent of the above measurements $[12,13]$ used approximately $1 \mathrm{fb}^{-1}$ of data. Although this represents only about one tenth of the anticipated final Tevatron data set, the precision of these measurements was already limited by experimental systematic uncertainties in the corrections for event selection efficiencies and unfolding of lepton momentum resolution. In order to unfold measured distributions for experimental resolution it is important that the chosen bin widths are not too small compared to the experimental resolution. In the low $Q_{T}$ region in $[12,13]$, the minimum bin sizes were determined by experimental resolution rather than the available data statistics. The final Tevatron data set will be an order of magnitude larger than that analysed in $[12,13]$. New ideas are therefore needed in order to exploit fully the data for studying the physics of boson $Q_{T}$.

The UA2 Collaboration introduced an alternative variable for studying the $Q_{T}$, which projects the $Q_{T}$ onto the bisector of the lepton pair in the transverse plane [14]. In the region of low $Q_{T}$, this reduces sensitivity to lepton momentum mis-measurement. A similar variable, $a_{T}$, was introduced in [15] and was proposed as an optimal variable for studying the $Q_{T}$ in [16]. Figure 1 illustrates this and other relevant variables defined below. Events with $\Delta \phi>\pi / 2$, where $\Delta \phi$ is the azimuthal opening angle of the lepton pair, correspond to approximately $99 \%$ of the total cross section. For such events the $Q_{T}$ is split into 


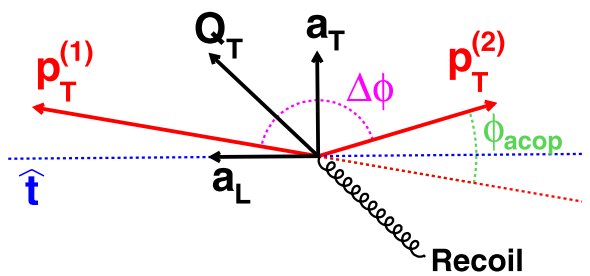

Fig. 1 Graphical illustration in the plane transverse to the beam direction of the variables defined in the text and used to analyse dilepton transverse momentum distributions in hadron colliders

two components with respect to an event axis defined as, $\hat{\mathbf{t}}=\left(\mathbf{p}_{T}{ }^{(1)}-\mathbf{p}_{T}{ }^{(2)}\right) /\left|\mathbf{p}_{T}{ }^{(1)}-\mathbf{p}_{T}{ }^{(2)}\right|$, where $\mathbf{p}_{T}{ }^{(1)}$ and $\mathbf{p}_{T}{ }^{(2)}$ are the lepton momentum vectors in the plane transverse to the beam direction. The component transverse to the event axis is denoted by $a_{T}$ and the aligned component is denoted by $a_{L}$. For events with $\Delta \phi<\pi / 2$ this decomposition is not useful and $a_{T}$ and $a_{L}$ are defined as being equal to $Q_{T}$. Compared to the $Q_{T}, a_{T}$ is less susceptible to the lepton $p_{T}$ resolution. In addition, the efficiencies of selection cuts on lepton isolation and $p_{T}$ are shown in [16] to be less correlated with $a_{T}$ than $Q_{T}$. For studying the low $Q_{T}$ (non-perturbative) region, $a_{T}$ is thus a more powerful variable than $Q_{T}$. The $a_{T}$ distribution has subsequently been calculated to NLL accuracy using soft gluon resummation techniques [17]. Recently, the $Q_{T}$ distribution has also been calculated at NNLL accuracy [18].

A recent paper [19] has discussed the idea of using $\Delta \phi$, as an analysing variable that is sensitive to the physics of $Q_{T}$, and insusceptible to lepton momentum uncertainties $^{1}$. Whilst $\Delta \phi$ is primarily sensitive to the same component of $Q_{T}$ as $a_{T}$, the translation from $a_{T}$ to $\Delta \phi$ depends on the scattering angle $\theta^{*}$ of the leptons relative to the beam direction in the dilepton rest frame. Thus, $\Delta \phi$ is less directly related to $Q_{T}$ than $a_{T}$. As a result, $\Delta \phi$ has somewhat smaller statistical sensitivity to the underlying physics than $a_{T}$, as will be demonstrated in this paper.

In this paper, we discuss two further ideas to improve experimental precision, whilst maintaining $\left(Q_{T}\right)$ physics sensitivity:

- Dividing $a_{T}$, and $Q_{T}$ by the dilepton invariant mass, $Q$, thus further reducing the effects of lepton $p_{T}$ resolution, and almost totally cancelling lepton $p_{T}$ scale calibration uncertainties.

- Correcting $\Delta \phi$ on an event-by-event basis for the scattering angle, $\theta^{*}$, thus improving the sensitivity to $Q_{T}$.

\footnotetext{
${ }^{1}$ We note that the expected distribution of $\Delta \phi$ does have a small residual sensitivity to the lepton $p_{T}$ measurement. This arises from the cut on $Q$ in the event sample selection, which is affected by the lepton $p_{T}$ scale and resolution.
}

An overview of the rest of this paper is as follows. In Sect. 2 we give an approximate analytic motivation for the idea of dividing $a_{T}$ (and $Q_{T}$ ) by $Q$ in order to produce variables with very substantially improved experimental resolution. In Sect. 3 we discuss the idea of correcting $\Delta \phi$ on an event-by-event basis for the scattering angle, $\theta^{*}$, thus improving the sensitivity to $Q_{T}$. In addition, we propose a new variable, $\cos \left(\theta_{\eta}^{*}\right)$, which provides a measure of the scattering angle that is based entirely on the measured track directions and is thus extremely well measured experimentally. In Sect. 4 we describe the simple parameterised detector simulation we employ in our MC studies. In Sect. 5-9 we present the results of our MC studies of the performance of the various candidate variables in terms of their experimental resolution and their sensitivity to the underlying physics. In Sect. 10 we present some conclusions, including our recommendations for the best variables to use in experimental studies of the transverse momentum of dilepton pairs produced at hadron colliders.

\section{Mass ratios of $a_{T}$ and $Q_{T}$}

For $\Delta \phi \approx \pi, a_{T}$ is given by the approximate formula

$a_{T}=2 \frac{p_{T}^{(1)} p_{T}^{(2)}}{p_{T}^{(1)}+p_{T}^{(2)}} \sin \Delta \phi$

and thus the fractional change in $a_{T}$ with respect to a variation in, say, $p_{T}^{(1)}$ is given by

$$
\frac{\Delta a_{T}}{a_{T}}=\frac{p_{T}^{(2)}}{p_{T}^{(1)}+p_{T}^{(2)}} \frac{\Delta p_{T}^{(1)}}{p_{T}^{(1)}} .
$$

The dilepton invariant mass is given by

$$
Q=\sqrt{2 p^{(1)} p^{(2)}(1-\cos \Delta \theta)},
$$

where $p^{(1)}$ and $p^{(2)}$ are the lepton momenta and $\Delta \theta$ is the angle between the two leptons. Thus, the fractional change in mass with respect to a variation in $p^{(1)}$ is given by

$$
\frac{\Delta Q}{Q}=\frac{1}{2} \frac{\Delta p^{(1)}}{p^{(1)}}
$$

Since track angles are extremely well measured it can be taken to a very good approximation that

$$
\frac{\Delta p_{T}^{(1)}}{p_{T}^{(1)}}=\frac{\Delta p^{(1)}}{p^{(1)}} .
$$

The fractional change in $a_{T} / Q$ with respect to a variation in $p^{(1)}$ is thus given by 
$\frac{\Delta\left(a_{T} / Q\right)}{\left(a_{T} / Q\right)}=\frac{\Delta a_{T}}{a_{T}}-\frac{\Delta Q}{Q}=\left(\frac{p_{T}^{(2)}}{p_{T}^{(1)}+p_{T}^{(2)}}-\frac{1}{2}\right) \frac{\Delta p_{T}^{(1)}}{p_{T}^{(1)}}$.

Thus the variations with $p_{T}^{(1)}$ in $a_{T}$ and $Q$ partially cancel in the ratio, rendering $a_{T} / Q$ less susceptible to the effects of lepton $p_{T}$ resolution than $a_{T}$. In particular, in the region of low $Q_{T}$ then $p_{T}^{(1)} \approx p_{T}^{(2)}$ and thus $\Delta\left(a_{T} / Q\right) \approx 0$. Similarly, the quantity $Q_{T} / Q$ is less susceptible to the effects of lepton $p_{T}$ resolution than $Q_{T}$.

A simple example of an uncertainty in the lepton $p_{T}$ scale calibration is to consider the $p_{T}$ of all leptons to be multiplied by a constant factor. It can be seen trivially that in this case $a_{T}, Q_{T}$ and $Q$ are all multiplied by the same factor and that the measured $a_{T} / Q$ and $Q_{T} / Q$ are unaffected by such a scale uncertainty.

\section{Correcting $\Delta \phi$ for the scattering angle}

The azimuthal opening angle between the two leptons, $\Delta \phi$, is primarily sensitive to the same component of $Q_{T}$ as $a_{T}$, and is based only on the well measured lepton angles. However, at fixed $a_{T} / Q, \Delta \phi$ depends on the scattering angle $\theta^{*}$ of the leptons relative to the beam direction in the dilepton rest frame. For convenience, we define the acoplanarity angle, $\phi_{\text {acop }}$, as $\phi_{\text {acop }}=\pi-\Delta \phi$. For $p_{T}^{(1)} \approx p_{T}^{(2)}$ it can be fairly easily shown that

$a_{T} / Q \approx \tan \left(\phi_{\text {acop }} / 2\right) \sin \left(\theta^{*}\right)$.

This suggests that the variable

$\phi^{*} \equiv \tan \left(\phi_{\text {acop }} / 2\right) \sin \left(\theta^{*}\right)$

may be an appropriate alternative quantity with which to study $Q_{T}$.

In the analysis of hadron-hadron collisions, $\theta^{*}$ is commonly evaluated in the Collins Soper frame [20]. However, $\theta_{\mathrm{CS}}^{*}$ requires knowledge of the lepton momenta and is thus susceptible to the effects of lepton momentum resolution. Motivated by the desire to obtain a measure of the scattering angle that is based entirely on the measured track directions (since this will give the best experimental resolution) we propose here an alternative definition of $\theta^{*}$. We apply a Lorentz boost along the beam direction such that the two leptons are back-to-back in the $r-\theta$ plane. This Lorentz boost corresponds to $\beta=\tanh \left(\frac{\eta^{-}+\eta^{+}}{2}\right)$ and yields the result ${ }^{2}$

$\cos \left(\theta_{\eta}^{*}\right)=\tanh \left(\frac{\eta^{-}-\eta^{+}}{2}\right)$,

\footnotetext{
${ }^{2}$ The lepton pseudorapidity, $\eta$, is defined as $\eta=-\ln \left[\tan \left(\frac{\theta}{2}\right)\right]$, where $\theta$ is the polar angle with respect to the beam direction, in the laboratory frame.
}

where $\eta^{-}$and $\eta^{+}$are the pseudorapidities of the negatively and positively charge lepton, respectively.

We consider two candidate variables

$$
\begin{aligned}
& \phi_{\mathrm{CS}}^{*} \equiv \tan \left(\phi_{\text {acop }} / 2\right) \sin \left(\theta_{\mathrm{CS}}^{*}\right), \\
& \phi_{\eta}^{*} \equiv \tan \left(\phi_{\text {acop }} / 2\right) \sin \left(\theta_{\eta}^{*}\right)
\end{aligned}
$$

for further evaluation in terms of their experimental resolution and physics sensitivity.

\section{Simple parameterised detector simulation}

Monte Carlo events are generated using PYTHIA [21], for the process $p \bar{p} \rightarrow Z / \gamma^{*}$, at $\sqrt{s}=1.96 \mathrm{TeV}$, in the $e^{+} e^{-}$and $\mu^{+} \mu^{-}$decay channels, and re-weighted in dilepton $Q_{T}$ and rapidity, $y$, to match the higher order predictions of RESBOS [22] as in [16]. Electrons and muons are defined at "particle level" according to the prescription in [23], and at "detector level" by applying simple Gaussian smearing to the particle level momenta: $\delta\left(1 / p_{T}\right)=3 \times 10^{-3}(1 / \mathrm{GeV})$ for muons, which are measured in the tracking detectors; $\delta p / p=0.4\left(p / p_{0}\right)^{-1 / 2}$ with $p_{0}=1 \mathrm{GeV}$ for electrons, which are measured in the calorimeter. In addition, the particle angles are smeared, assuming Gaussian resolutions of $0.3 \times 10^{-3} \mathrm{rad}$ for $\phi$ and $1.4 \times 10^{-3}$ for $\eta$. These energy, momentum, and angular resolutions roughly correspond to those in the DØ detector [24].

Events are accepted for further analysis if: $70<Q<$ $110 \mathrm{GeV}$ and both leptons satisfy the requirements $p_{T}>$ $15 \mathrm{GeV}$ and $|\eta|<2$. These selection cuts are made at particle level, unless otherwise stated.

\section{Scaling factors}

In the following sections, we compare the experimental resolution and physics sensitivity of the various candidate variables. In particular, we compare the variation of the resolution for each variable as a function of that variable. This comparison is facilitated by ensuring that all distributions have approximately the same scale and shape. Compared to $Q_{T}$ or $Q_{T} / Q$, all other variables are on average a factor $\sqrt{2}$ smaller (since $a_{T}$ and $a_{L}$ measure one component of $\left.Q_{T}\right)$. A simple multiplication by $M_{Z}(=91.19 \mathrm{GeV}$ [25]) corrects for the average $Q^{-1}$ factor in the mass ratio and angular variables and conveniently ensures that all variables have the same units $(\mathrm{GeV})$. Finally, the mean value of $\sin \left(\theta^{*}\right)$ is around $\sim 0.85$, and $\tan \left(\phi_{\text {acop }} / 2\right)$ is scaled by this additional factor. The above factors are summarised in Table 1. 


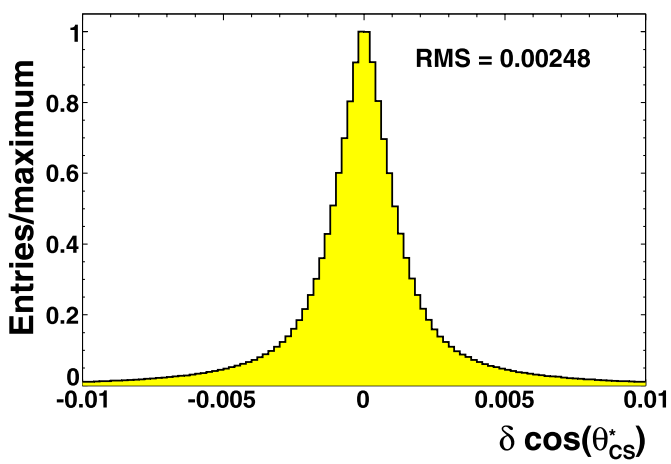

(a) $70<Q<110 \mathrm{GeV}$

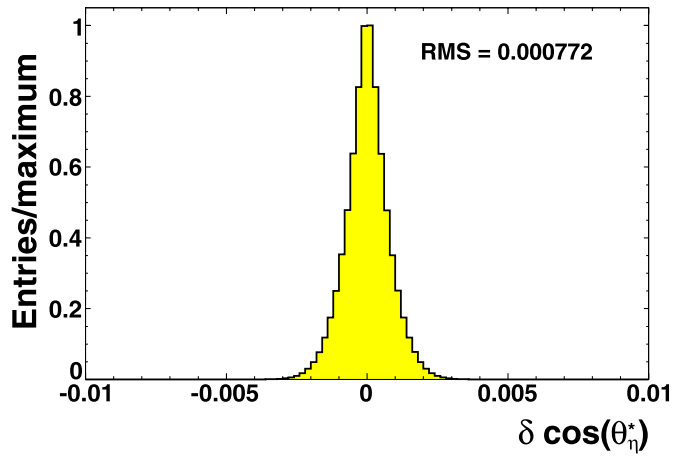

(b) $70<Q<110 \mathrm{GeV}$

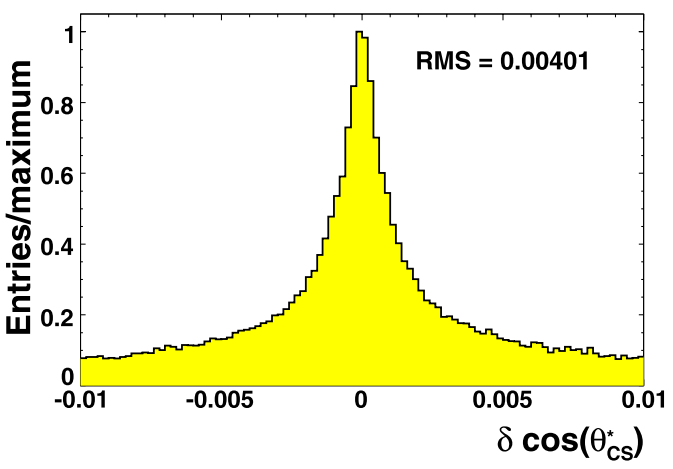

(c) $500<Q<600 \mathrm{GeV}$

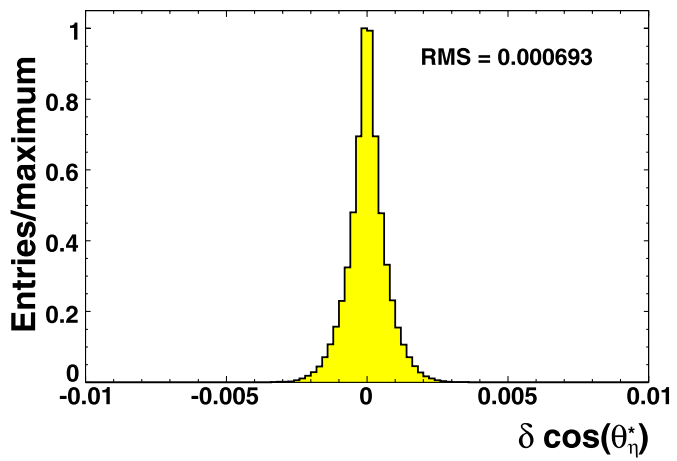

(d) $500<Q<600 \mathrm{GeV}$

Fig. 2 Distributions of experimental resolutions obtained for the dilepton centre of mass scattering angle for events satisfying $70<Q<110 \mathrm{GeV}$ (top) and $500<Q<600 \mathrm{GeV}$ (bottom). a and $\mathbf{c}$ show $\cos \left(\theta_{\mathrm{CS}}^{*}\right)$. b and $\mathbf{d}$ show $\cos \left(\theta_{\eta}^{*}\right)$
Table 1 Scaling factors for different candidate variables

\begin{tabular}{ll}
\hline Variable & Scaling factor \\
\hline$Q_{T}$ & 1 \\
$Q_{T} / Q$ & $M_{Z}$ \\
$a_{T}$ & $\sqrt{2}$ \\
$a_{T} / Q$ & $\sqrt{2} M_{Z}$ \\
$a_{L}$ & $\sqrt{2}$ \\
$a_{L} / Q$ & $\sqrt{2} M_{Z}$ \\
$\tan \left(\phi_{\mathrm{acop}} / 2\right)$ & $0.85 \sqrt{2} M_{Z}$ \\
$\phi_{\mathrm{CS}}^{*}$ & $\sqrt{2} M_{Z}$ \\
$\phi_{\eta}^{*}$ & $\sqrt{2} M_{Z}$ \\
\hline
\end{tabular}

\section{Experimental resolution for dilepton scattering angle}

Figure 2 shows the experimental resolution of $\cos \left(\theta_{\mathrm{CS}}^{*}\right)$ and $\cos \left(\theta_{\eta}^{*}\right)$ in our simulation of dimuon events. The upper row of Fig. 2 shows events that satisfy $70<Q<110 \mathrm{GeV}$; it demonstrates that $\cos \left(\theta_{\eta}^{*}\right)$ is significantly better measured experimentally than $\cos \left(\theta_{\mathrm{CS}}^{*}\right)$. This is because $\cos \left(\theta_{\eta}^{*}\right)$ is evaluated using only angular measurements, which are more precise than the momentum measurements included in the determination of $\cos \left(\theta_{\mathrm{CS}}^{*}\right)$.

The variable $\cos \left(\theta_{\eta}^{*}\right)$ is used in the definition of $\phi_{\eta}^{*}=$ $\tan \left(\phi_{\text {acop }} / 2\right) \sin \left(\theta_{\eta}^{*}\right)$ in Sect. 3 above. As an aside, we note in addition that a precise determination of the dilepton centre of mass scattering angle that is free from the effects of lepton momentum resolution can also find application in the determination of the forward-backward charge asymmetry of dilepton production at hadron colliders. The experimental resolution in $\cos \left(\theta_{\mathrm{CS}}^{*}\right)$ becomes particularly significant in the dimuon channel for very high values of $Q$ for which the lepton momentum resolution is poorest. This is illustrated in the lower row of Fig. 2, which shows the experimental resolution of $\cos \left(\theta_{\mathrm{CS}}^{*}\right)$ and $\cos \left(\theta_{\eta}^{*}\right)$ in events that satisfy $500<Q<600 \mathrm{GeV}$. The advantage of using $\cos \left(\theta_{\eta}^{*}\right)$ for high mass events is even larger than was the case for $70<Q<110 \mathrm{GeV}$.

\section{Experimental resolution for variables related to dilepton $Q_{T}$}

Figure 3 compares separately for our dimuon and dielectron simulations, the resolution of various candidate variables, as a function of that variable (at particle level). All variables are scaled by the factors in Table 1 .

The following observations are made:

- $a_{T} / Q$ is significantly better measured than $a_{T}$, over the entire range.

- Similarly, $Q_{T} / Q$ is significantly better measured than $Q_{T}$. 


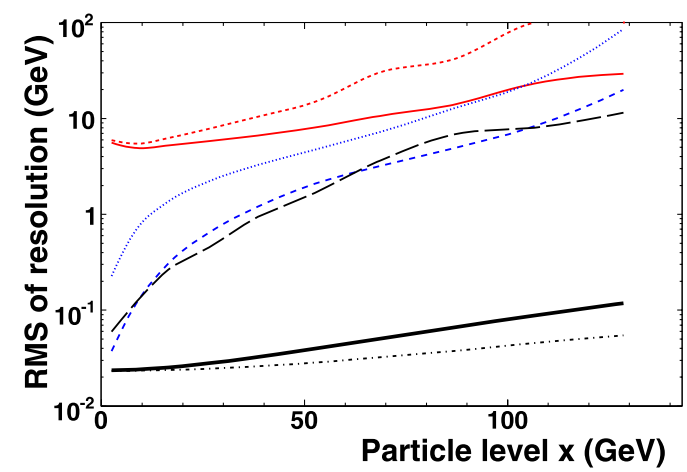

(a) Tracker

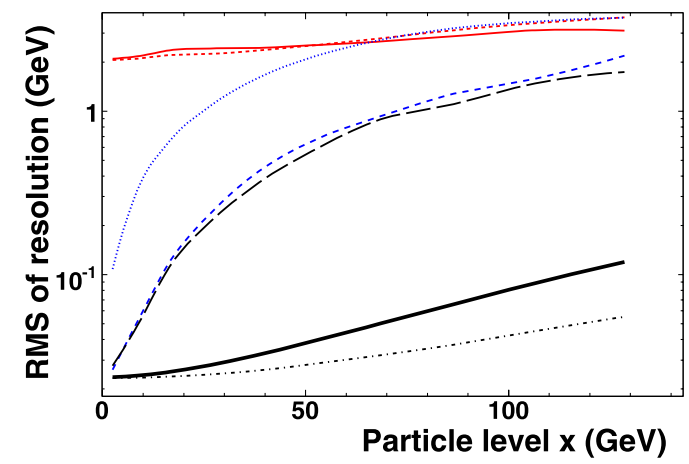

(b) Calorimeter

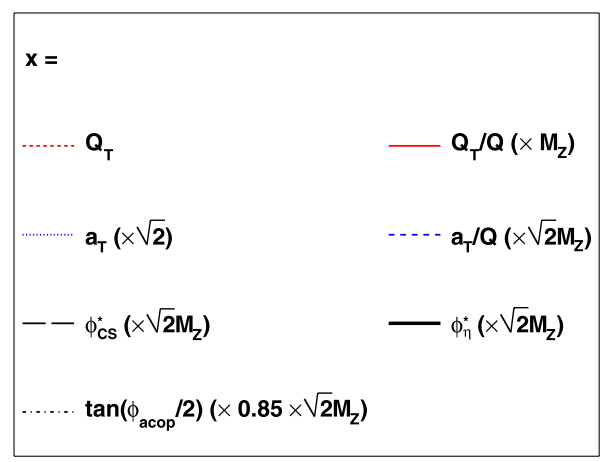

Fig. 3 The RMS resolution of each candidate variable, as a function of that variable (scaled by the factors described in the text). Results are presented both for $\mathbf{a}$ muons (tracker) and $\mathbf{b}$ electrons (calorimeter) resolution in the lepton momenta

- Over the full range, $a_{T}$ and $a_{T} / Q$ perform better than $Q_{T}$ and $Q_{T} / Q$ respectively.

- Compared to $a_{T} / Q, \phi_{\mathrm{CS}}^{*}$ has similar resolution, while $\phi_{\eta}^{*}$ is significantly better.

- The most precisely measured variable is $\tan \left(\phi_{\text {acop }} / 2\right)$, since it is determined only from the azimuthal angles of the leptons, whereas the uncertainty on $\phi_{\eta}^{*}$ includes also the uncertainties on the measured lepton pseudorapidities.

Since the discussion in Sect. 2 is only approximate, we have investigated empirically various other possible scalings of $a_{T}$ with $Q$ (with the appropriate scale factor applied, as above). Irrespective of whether tracker-like or calorimeter-like resolution in the lepton momenta is simulated, $a_{T} / Q$ is verified to have the best experimental resolution for all $Q_{T}$.

\section{Event selection efficiency}

Most future hadron collider analyses of $Z / \gamma^{*} \rightarrow l^{+} l^{-}$ events will require the leptons to be isolated in order to control the backgrounds from heavy flavour decays. In addition, requirements on calorimeter shower shape, which are needed to control background from jets mis-reconstructed as electrons, are likely to have an efficiency that depends on the degree to which the leptons are isolated. For example, recent measurements of the $Z / \gamma^{*}$ inclusive cross section by the ATLAS [26] and CMS [27] experiments imposed requirements on lepton isolation and/or calorimeter shower shape, similarly to the Tevatron analyses. Efficiencies for such cuts depend strongly on the $Q_{T}$, and as discussed in [16] these dependencies result mostly from the $a_{L}$ component of the $Q_{T}$. A large $a_{L}$ component corresponds to a high transverse momentum hadronic recoil, with a potentially large fraction projected onto one of the leptons. Compared to an event with smaller $a_{L}$, it is less likely for both leptons to satisfy isolation requirements. In [16], $a_{T}$ was shown to be less correlated with isolation efficiencies than the $Q_{T}$. We have verified that any of the variables proposed here, which are primarily sensitive to the $a_{T}$ component of the $Q_{T}$, exhibit comparable efficiency dependencies to those of $a_{T}$ itself. Thus, on these grounds, we find no disadvantage of e.g. $\phi_{\eta}^{*}$ or $a_{T} / Q$, compared to $a_{T}$.

\section{Sensitivity to the physics}

The width of the $Q_{T}$ distribution is expected to scale like $\ln (Q)$ [3]. Figure 4 shows the particle level, normalised distributions of $a_{T}, M_{Z} a_{T} / Q$ and $\ln \left(M_{Z} / Q_{0}\right) a_{T} / \ln \left(Q / Q_{0}\right)$ (with $Q_{0}=1 \mathrm{GeV}$ ) for three ranges of $Q$. We see that $a_{T}$ has a mild dependence on $Q$, while dividing by $Q$ over corrects this dependence. In this respect, we observe that the distribution in $a_{T} / \ln \left(Q / Q_{0}\right)$ has the smallest dependence on $Q$, as might be expected from [3].

Experimental measurements of $Z$ boson production are typically made over a fairly wide bin in $Q$ (e.g., 70$110 \mathrm{GeV})$. One potential concern with measurements of $a_{T} / Q$ and $Q_{T} / Q$ is that the increased correlation with $Q$ demonstrated in Fig. 4 might degrade the sensitivity to the underlying physics. Since $\phi_{\eta}^{*}$ behaves approximately as $\phi_{\eta}^{*} \approx a_{T} / Q$, a similar degradation in the physics sensitivity of $\phi_{\eta}^{*}$ may be expected. In this respect, $a_{T} / \ln \left(Q / Q_{0}\right)$ is a more suitable variable than $a_{T} / Q$ for studying the boson $Q_{T}$ distribution over a wide range in $Q$. However it has poorer experimental resolution, as was discussed in Sect. 7. 

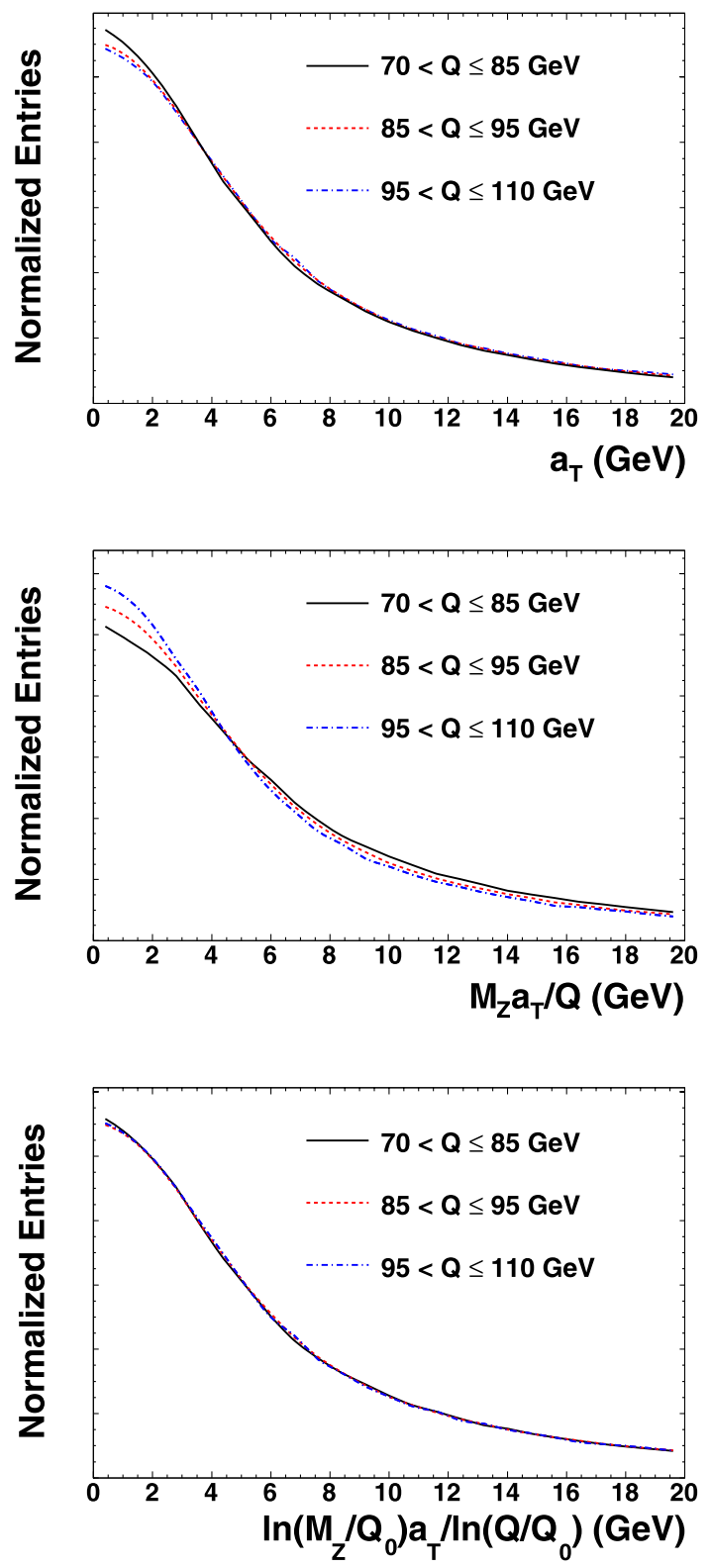

Fig. 4 Comparison of particle level distributions of $a_{T}, M_{Z} a_{T} / Q$ and $\ln \left(M_{Z} / Q_{0}\right) a_{T} / \ln \left(Q / Q_{0}\right)$ for three ranges of $Q$

Using a similar procedure to that described in [16], we study the sensitivity to the underlying physics of the different candidate variables. We run pseudo-experiments to fit for the value of the parameter $g_{2}$, which determines the width of the low $Q_{T}$ region in RESBOS. Events must meet the requirements described in Sect. 4, except that these are applied at detector level rather than particle level. Of these events, $1 \mathrm{M}$ are assigned as pseudo-data and the remaining events are used to build $g_{2}$ templates. All variables are scaled by the factors listed in Table 1, such that the same binning (30 equal width bins in the range $0-30 \mathrm{GeV}$ ) can be used. A minimum $\chi^{2}$ fit determines the statistical sensitivity of each variable to the value of $g_{2}$.
Table 2 Statistical sensitivity (in \%) on the parameter $g_{2}$ from fits to the distributions of different variables. For details see text

\begin{tabular}{llll}
\hline Variable & Particle level & Calorimeter & Tracker \\
\hline$Q_{T}$ & 0.65 & 0.94 & 1.41 \\
$Q_{T} / Q$ & 0.65 & 0.94 & 1.40 \\
$a_{T}$ & 1.00 & 1.00 & 1.00 \\
$a_{T} / Q$ & 1.00 & 1.01 & 1.00 \\
$a_{L}$ & 1.21 & 2.35 & 4.74 \\
$\tan \left(\phi_{\mathrm{acop}} / 2\right)$ & 1.04 & 1.05 & 1.04 \\
$\phi_{\mathrm{CS}}^{*}$ & 1.00 & 1.00 & 0.99 \\
$\phi_{\eta}^{*}$ & 1.00 & 1.00 & 0.99 \\
\hline
\end{tabular}

Table 3 Statistical uncertainty (in $\%$ ) on the parameter $K_{Q_{T}}$ (as defined in the text) from fits to the distributions of different variables. For details see text

\begin{tabular}{llll}
\hline Variable & Particle level & Calorimeter & Tracker \\
\hline$Q_{T}$ & 1.65 & 1.67 & 1.82 \\
$Q_{T} / Q$ & 1.66 & 1.67 & 1.78 \\
$a_{T}$ & 1.92 & 1.92 & 1.96 \\
$a_{T} / Q$ & 1.92 & 1.92 & 1.94 \\
$a_{L}$ & 1.98 & 2.02 & 2.34 \\
$\tan \left(\phi_{\mathrm{acop}} / 2\right)$ & 1.96 & 1.96 & 1.98 \\
$\phi_{\mathrm{CS}}^{*}$ & 1.88 & 1.88 & 1.90 \\
$\phi_{\eta}^{*}$ & 1.87 & 1.87 & 1.92 \\
\hline
\end{tabular}

Whilst in [16] we were mostly interested in the low $Q_{T}$ region, the ideas proposed in this paper also improve experimental resolution at higher $Q_{T}$ (see Sect. 7). Thus it is of interest to study the physics sensitivity also in this region. We similarly fit for a parameter $K_{Q_{T}}$, which weights events with (particle level) $Q_{T}>25 \mathrm{GeV}$, by $K_{Q_{T}}\left(Q_{T}-25\right)$, and approximately represents the differences between predictions at NLO and NNLO discussed in [12]. Again, after applying the appropriate scaling factors from Table 1, the same binning can be used for each variable ${ }^{3}$.

The results of the fits to $g_{2}$ and $K_{Q_{T}}$ are presented in Tables 2 and 3 respectively. Results are given separately for particle-level (dimuon) and detector-level (tracker and calorimeter). For both $a_{T} / Q$ and $Q_{T} / Q$, the statistical sensitivities are essentially the same as those for $a_{T}$ and $Q_{T}$ respectively. Thus the effect of the additional $Q$ dependence is shown to be negligible.

The approximately $5 \%$ poorer sensitivity of $\tan \left(\phi_{\text {acop }} / 2\right)$, compared to $a_{T} / Q$, demonstrates the $\sin \left(\theta^{*}\right)$ ambiguity of the former. The additional factor $\sin \left(\theta^{*}\right)$, present in $\phi_{\eta}^{*}$

\footnotetext{
${ }^{3}$ The first bin is of width $5 \mathrm{GeV}$ (with lower edge at zero) and each consecutive bin is $2 \mathrm{GeV}$ wider than the last. Ten such bins give an upper edge of the last bin at $140 \mathrm{GeV}$ and the fit includes the overflow bin from $140 \mathrm{GeV}$ to $\infty$.
} 
and $\phi_{\mathrm{CS}}^{*}$ actually recovers the sensitivity to the same level as $a_{T}$. In addition, the $\phi_{\eta}^{*}$ variable, which was shown in Sect. 7 to have the best experimental resolution (except for $\left.\tan \left(\phi_{\text {acop }} / 2\right)\right)$, performs similarly to $\phi_{\mathrm{CS}}^{*}$ in terms of physics sensitivity.

Of course, the results presented in Tables 2 and 3 represent only the statistical sensitivity of the considered variables when compared to $Q_{T}$. As discussed in [16], the systematic uncertainties associated with modelling of detector resolution and efficiency will be reduced using variables that are better measured and less correlated with event selection efficiencies than is the case for $Q_{T}$.

\section{Summary and conclusions}

Measurements of dilepton $\left(Z / \gamma^{*}\right)$ transverse momentum, $Q_{T}$, distributions are crucial for improving models of vector boson production at hadron colliders. The precision of future measurements of the $Q_{T}$ distribution at the Tevatron and LHC will be totally limited by uncertainties in correcting for detector resolution and efficiency, and the minimum bin sizes will be limited by resolution.

In [16] an alternative variable, $a_{T}$, was demonstrated to be significantly less susceptible to such detector effects than $Q_{T}$. We have shown in this article that the experimental resolution of $a_{T}$ can be further improved by taking the ratio to the measured dilepton invariant mass, $Q$. Similarly, we have demonstrated that the variable $Q_{T} / Q$ is experimentally more precisely measured than $Q_{T}$. No obvious disadvantages of the variable $a_{T} / Q\left(Q_{T} / Q\right)$ as compared to $a_{T}$ $\left(Q_{T}\right)$ are found when studying the efficiency dependencies or the physics sensitivity (by making fits to parameters describing the shape of the $Q_{T}$ distribution).

The acoplanarity angle, $\phi_{\text {acop }}$, is also sensitive to $Q_{T}$. However, it has the approximate dependence $a_{T} / Q \approx$ $\tan \left(\phi_{\text {acop }} / 2\right) \sin \left(\theta^{*}\right)$, where $\theta^{*}$ is the scattering angle of the leptons with respect to the beam direction in the dilepton rest frame. Thus $\phi_{\text {acop }}$ is less directly related to $Q_{T}$ than $a_{T}$. We show that correcting $\tan \left(\phi_{\text {acop }} / 2\right)$ by a factor $\sin \left(\theta^{*}\right)$ yields a variable with essentially the same statistical sensitivity as $a_{T} / Q$, whilst the experimental resolution is significantly better. Furthermore, using an approximate rest frame determined using only angular information, we propose a new method to measure the scattering angle, $\theta_{\eta}^{*}$, with the best possible experimental resolution. We conclude that in the region of low $Q_{T}$ the variable $\phi_{\eta}^{*} \equiv \tan \left(\phi_{\text {acop }} / 2\right) \sin \left(\theta_{\eta}^{*}\right)$ represents possibly the optimal combination of physics sensitivity, experimental resolution and immunity to experimental systematic uncertainties.

For studying the high $Q_{T}$ region, the optimal variable is $Q_{T} / Q$, which is significantly better measured than $Q_{T}$, and has no disadvantages in terms of physics sensitivity or efficiency dependence. However, it is interesting to study correlations between the $a_{T}$ and $a_{L}$ components of $Q_{T}$. Therefore, measurements of $\phi_{\eta}^{*}$ in the high $Q_{T}$ region will be complementary to measurements of $Q_{T} / Q$.

These further optimisations of variables used to study the $Q_{T}$ distribution will allow significantly finer binning and smaller unfolding corrections (and thus uncertainties), enabling tighter constraints on vector boson production models.

Open Access This article is distributed under the terms of the Creative Commons Attribution Noncommercial License which permits any noncommercial use, distribution, and reproduction in any medium, provided the original author(s) and source are credited.

\section{References}

1. K. Melnikov, F. Petriello, Phys. Rev. D 74, 114017 (2006)

2. S. Catani et al., Phys. Rev. Lett. 103, 082001 (2009)

3. J. Collins, D. Soper, G. Sterman, Nucl. Phys. B 250, 199 (1985)

4. F. Landry et al., Phys. Rev. D 67, 073016 (2003)

5. T. Sjöstrand, S. Mrenna, P. Skands, J. High Energy Phys. 05, 026 (2006)

6. G. Corcella et al., J. High Energy Phys. 0101, 010 (2001)

7. M.L. Mangano et al., J. High Energy Phys. 0307, 001 (2003)

8. T. Gleisberg et al., J. High Energy Phys. 0902, 007 (2009)

9. T. Affolder et al. (CDF Collaboration), Phys. Rev. Lett. 84, 845 (2000)

10. B. Abbott et al. (DØ Collaboration), Phys. Rev. D 61, 032004 (2000)

11. B. Abbott et al. (DØ Collaboration), Phys. Rev. Lett. 84, 2792 (2000)

12. V.M. Abazov et al. (DØ Collaboration), Phys. Rev. Lett. 100, $102002(2008)$

13. V.M. Abazov et al. (D0 Collaboration), Phys. Lett. B 693, 522 (2010)

14. J. Alitti et al. (UA2 Collaboration), Z. Phys. C 47, 523 (1990)

15. K. Ackerstaff et al. (OPAL collaboration), Eur. Phys. J. C 4, 47 (1998)

16. M. Vesterinen, T.R. Wyatt, Nucl. Instrum. Methods Phys. Res., Sect. A, Accel. Spectrom. Detect. Assoc. Equip. 602, 432 (2009)

17. A. Banfi, M. Dasgupta, R.M. Duran Delgado, J. High Energy Phys. 0912, 022 (2009)

18. G. Bozzi et al. (2010). arXiv:1007.2351v1 [hep-ph]

19. M. Boonekamp, M. Schott (2010). arXiv:1002.1850v1 [hep-ex]

20. J. Collins, D. Soper, Phys. Rev. D 16, 2219-2225 (1977)

21. T. Sjöstrand et al., Comput. Phys. Commun. 135, 238 (2001)

22. C. Balazs, C.P. Yuan, Phys. Rev. D 56, 5558-5583 (1997)

23. J.M. Butterworth et al. (2010). arXiv:1003.1643v1 [hep-ph]

24. V.M. Abazov et al. (DØ Collaboration), Nucl. Instrum. Methods Phys. Res., Sect. A, Accel. Spectrom. Detect. Assoc. Equip. 565, 465-537 (2006)

25. C. Amsler et al., Phys. Lett. B 667, 1 (2008)

26. G. Aad et al. (ATLAS Collaboration), J. High Energy Phys. 1012, $060(2010)$

27. V. Khachatryan et al. (The CMS Collaboration), J. High Energy Phys. 1101, 080 (2011) 